\title{
SEISMIC ACTIVITY SEEN THROUGH EVOLUTION OF THE HURST EXPONENT MODEL IN 3D
}

\author{
J. PATIÑO ORTIZ, ${ }^{*} \ddagger$ R. CARREÑO AGUILERA, ${ }^{*}$ \\ A. S. BALANKIN, ${ }^{*}$ M. PATIÑO ORTIZ, ${ }^{*}$ \\ J. C. TOVAR RODRIGUEZ, ${ }^{*}$ M. A. ACEVEDO MOSQUEDA,* \\ M. A. MARTINEZ CRUZ* and WEN YU ${ }^{\dagger}$ \\ *Fractal Mechanical Group, Instituto Politécnico Nacional \\ Zacatenco. Av. IPN S/N. Col. Lindavista. 07738. Mexico, F. D. \\ ${ }^{\dagger}$ Cinvestav, Instituto Politécnico Nacional \\ Zacatenco. Av. IPN S/N. Col. Lindavista. 07738. Mexico, F. D. \\ †Jpatinoo@ipn.mx
}

Received June 14, 2014

Accepted September 12, 2016

Published October 13, 2016

\begin{abstract}
The dynamics seismic activity occurred in the Cocos Plate-Mexico is analyzed through the evolution of Hurst exponent and 3D fractal dimension, under the mathematical fractal structure based on seismic activity time series, taking into account the magnitude $(M)$ as the main parameter to be estimated. The seismic activity time series and, annual intervals are considered first for finding the Hurst exponent of each year since 1988 (the year in which the database is consistent) until 2012, and then the following years are accumulated describing the cumulative Hurst exponent. The seismic activity description is based on Cocos Plate data information; during a period conform from 1 January 1988 to 31 December 2012. Analyses were performed following methods, mainly considering that the Hurst exponent analysis provides the ability to find the seismicity behavior time-space, described by parameters obtained under the fractal dimension and complex systems.
\end{abstract}

Keywords: Hurst Coefficient; Fractals; Seismic Activity; Time Series.

This is an Open Access article published by World Scientific Publishing Company. It is distributed under the terms of the Creative Commons Attribution 4.0 (CC-BY) License. Further distribution of this work is permitted, provided the original work is properly cited. 


\section{INTRODUCTION}

The seismicity is a complex natural phenomenon studied from its time-space characteristics, which is manifested according to certain statistical laws governing its occurrence. ${ }^{1-4}$ The correlations that may arise between in the dynamics earthquakes occurrence have been the subject of many studies conducted around the world, especially in the most affected countries. ${ }^{5-11}$ Recently, worldwide the research activity concerning the seismic phenomenon has focused on the study of correlations viewed in the time series space diagram. ${ }^{1-4}$

The study of seismic activity has been developed for smaller scales; however, the challenge is to find the seismic activity for larger scales of Richter considering the smaller results.

Turcotte $^{3}$ described that the earthquake epicenter is bounded by a region that follows a fractal distribution. So that, the scaling laws of temporal and spatial variability of earthquakes have been obtained, considering several seismic regions with different tectonic properties. ${ }^{5-8}$

However, despite of the best efforts developed around the world to know the seismic activity, specialists in the area are still working to understand its dynamics..$^{5-11}$

A worldwide challenge is the prediction of earthquakes, specifically for large earthquakes. It is possible from past seismic activity, or from other phenomena related to the seismic activity, known as precursors. ${ }^{12-14}$

The earthquakes in a given region have a fractal distribution 3, at least when they are viewed in two dimensions (epicenter). Fortunately, these results have a great similarity in different regions worldwide for similar Richter magnitudes. The fractal description indicates that seismicity is hierarchically organized presenting the same structure at different scales of observation, and raising the question: "Is it necessary to go beyond the GutenbergRichter law? ${ }^{9}$ Therefore, wondering whether the simple ratio contained in this law (about 100 earthquakes with $M \geq 5$, for every 10 with $M \geq 6$ and, for each with $M \geq 7$ ) is a reflection of a selfsimilarity deeper in the structure of seismicity. In order words, in a given specific region, Is it equivalent in a 1 year of earthquakes with $M \geq 5$, to 10 years with $M \geq 6$, or for 100 years with $M \geq 7$ ?, the answer to this question would have important implications for seismic hazard studies, and it would be a significant progress toward earthquake prediction as well. ${ }^{10-14}$

One of the most important parameters that arise when the seismicity is analyzed within the framework of fractal geometry is the Hurst exponent, giving the possibility of determining persistence conditions presented by the seismic phenomenon and also the variability of the fractal dimension of the hypo-centers (3D).

The main goal of this paper is to develop two analysis of the registered seismic data in the southwestern Mexico (Cocos Plate), in order to search in this way if there is a pattern of behavior of the considered variables (Hurst exponent and $D_{f}$ in $3 \mathrm{D}$ ) or if there is any relationship between their behavior. It also aims to find correlations that allow us to describe the seismic phenomenon through the behavior of the variables involved and their interaction. The main goal is also about to find relationships between the Hurst exponent and fractal dimension in $3 \mathrm{D}$ in the time series of seismic activity in order to understand their space-time behavior better such that we can study this space-time behavior in a closer way to the reality.

The organization of the document consists in four parts: Introduction, complex systems, development and conclusions. The introduction talks about the general topics about the analysis, concepts and main goal of the seismic activity, and challenges in this area, in Sec. 2 an overall about the complex systems, its importance and difficulty to do predictions for these systems, Sec. three is the core of this paper since it describes the development of the analysis of the hurt exponent evolution for the seismic activity in 3 dimension (3D), and the last section is just about the conclusions of this papers, saying about the result of the analysis of the 3D Hurst exponent evolution.

\section{FRACTAL ANALYSIS}

For many years, during the study of different phenomena, scientists have been unable to explain some situations in satisfactory sense. The possibility to develop predictions is a very important issue and it is common to different phenomena. For instance, if today is a raining day, then is it possible to predict whether it will rain tomorrow or day after tomorrow?, i.e. if we know what will happen in the future and where we stand now, we could establish the strategies required with a minimal cost. ${ }^{15}$ 
The main purpose of science is its ability to link causes and effects. The study of complex systems in the framework of the theory of fractals has been recognized in recent years as a new scientific discipline, one of the latest interdisciplinary fields.

A real complex system generally has an invariant scale, i.e. its behavior does not change by the rescaling of the variables that govern its dynamics. This gives the possibility of using the scaling approach for self-similar fractals and self-affine. ${ }^{16-18}$

The self-similar fractals remain invariant when the scale is changed uniformly in all directions. The invariance can be literal, and performing a zoom of a part is seen exactly the same structure, or may be a factor scale: it looks exactly the same, but the result of the zoom is indistinguishable from the original set. Any statistical estimator that applies to this set leads to the same results as if applied to the incomplete set.

However, it is easy to find situations where two or more directions are not equivalent. For example, in a fractal constructed by addition, one direction can be introduced if the particles reach the set from one direction only (deposition). In the whole growth set in this case there is no classic self-similar fractal, but belongs to a different type of fractals, called self-affine fractals.

The nature of any affinity transformation can be classified in two groups: linear and nonlinear. The fundamental difference between them lies in the fact that the first group, the straight lines which form the geometric shape are preserved, while in the second group this does not occur, therefore position, orientation and size are modified, as shown in Fig. $1{ }^{19}$

There are different methods for determining the properties of a fractal. They can be classified mainly as: (1) methods to determine the self-similar fractals characteristics and (2) methods to determine characteristics in self-affine fractals.

The fundamental difference between self-similarity and self-affinity fractals is the way in which scaling produces statistical equivalence.

Considering a function $Y$ of an independent variable $t$ (time, horizontal) and $Y$ as the dependent variable (vertical), the self-affinity is defined through statistical invariance under the following transformation:

$$
\begin{gathered}
t \rightarrow \lambda t, \\
Y \rightarrow \lambda^{H} Y .
\end{gathered}
$$

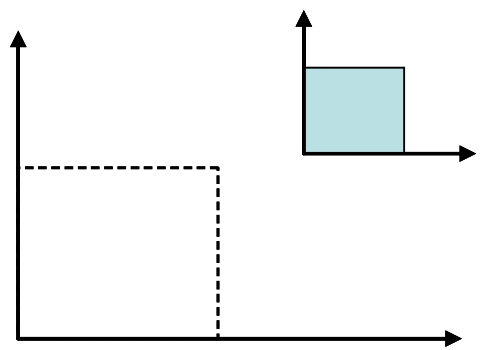

(a)

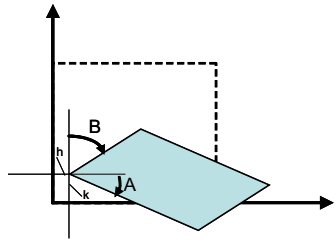

(b)
Fig. 1 Several transformations in: (a) a similarity transformation factor of $r=0.5$ and (b) affinity linear transformation, with rotation and translation. Source: Adapted from: Fractus, Fracta, fractal. ${ }^{19}$

The scaling parameter $H$ is described as the Hurst exponent. ${ }^{19}$

In many cases, spatial scaling behavior is attributed to the invariant statistics of the saturated interfaces under the transformation self- affine scaling $\left(\lambda x, \lambda^{\alpha} z\right)$. The self-affine invariance implies

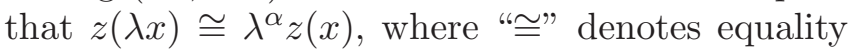
in the statistical sense, and no characteristic length scale at the interface compared to the size of the system. Therefore, in this case the magnitude of fluctuations in local heights on a window size $l<L$ remains saturated in time $t_{s} \sim L^{z}$, regardless of the size of the system, that is, $h\left(L, t>t_{s}\right) \sim L^{\varsigma}$, where $\varsigma=\alpha$ is the local roughness exponent. If so, the morphology of an interface of a crack is characterized by unique roughness exponent, $0 \leq \alpha=\varsigma \equiv$ $H \leq 1$, which is commonly called the Hurst exponent. ${ }^{20,21}$ The latter is related to the local dimension (2D fractal) of counting boxes of the interface as $D_{B}=2-H$.

Hurst exponent is also an indicator to determine if a feature or a time series exhibits fractal behavior and measures the intensity of long-term dependency of a time series. It is said that the phenomenon analyzed is random when $H=0.5$ (white noise) that is persistent when $0.5<H<1$ (there is scale invariance associated with long-term positive correlations), and is anti-persistent when $0<H<0.5$ (there is scale invariance associated with negative long-term correlations). ${ }^{22-24}$

\subsection{Rescaled Range Analysis}

The rescaled range method $(R / \sigma)$ proposed by Mandelbrot and Wallis ${ }^{25-27}$ based on a previous hydrological analysis of Hurst, ${ }^{28,29}$ is the oldest 


\section{J. Patiño Ortiz et al.}

and well-known method to determine the value of $H$. This method allows the calculation of the selfsimilarity parameter $H$, to measure the intensity of long-term dependence time series.

For a time series $X=\left\{X_{t}: t=1, n\right\}$ of length $n$; $R / \sigma$ is defined as the ratio between maximum range normalized integrated signal $R(n)$ with respect to the standard deviation $\sigma$ :

$$
\frac{R(n)}{\sigma(n)}=\frac{\max \left\{0, r_{t}: t=1, n\right\}-\min \left\{0, r_{t}: t=1, n\right\}}{(S(n))^{1 / 2}}
$$

with

$$
r_{k}=\sum_{t=1}^{k} X_{t}-\frac{k}{n} \sum_{t=1}^{n} X_{t}
$$

and

$$
S(n)=\left[\frac{1}{n} \sum_{t=1}^{n}\left(X_{t}-\frac{1}{n} \sum_{t=1}^{n} X_{t}\right)^{2}\right] .
$$

A reliable measurement of $S(n)$ or, $\sigma$, requires sampled data with constant interval $d_{n}$, since the expected difference between the constant values of $X$ is a function of the distance between them.

If the plot is self-affine, the expected value of $R / \sigma$ has a scaling $n^{H}$ when $n \rightarrow \infty$ :

$$
R / \sigma \propto \tau^{H} .
$$

The accuracy in the determination of $H$, in all time slots depends on the number of data which are used in the calculation. If this number is reasonably large (that is, when the maximum time interval is traced several times), it is expected that the curve $R / \sigma$ provides information on the self-similarity of all timeslots. If the recorded time has only short-term correlations, then the log-log plot is also a straight line with a slope of 0.5 . The major drawback of analyzes $R / \sigma$ is that the asymptotic distribution theory has been derived for the parameter $H$.

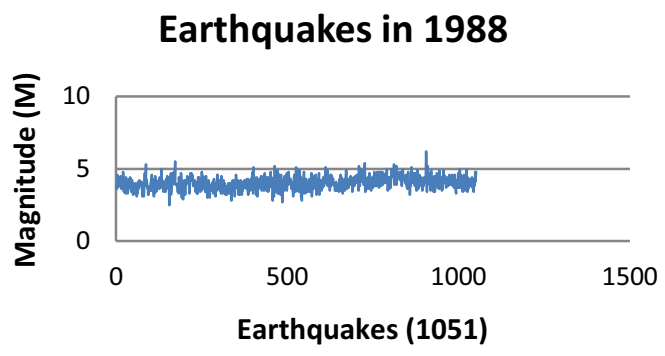

(a)

\section{DATA ANALYSIS DEVELOPMENT}

\subsection{Seismic Data}

In this research, data series of seismic events (by magnitude) occurred in the Cocos Plate-Mexico are used. These series correspond to a period from 1 January 1988 to 31 December 2012, between the coordinates: Lengths $-9^{\circ}$ and $-106^{\circ}(W)$; Latitudes $13^{\circ}$ and $19^{\circ}(N)$ (Fig. 2), with a total of 31,276 seismic events with magnitude $M \geq 3$, the data period is 25 years, beginning with the earthquake occurred on 01/01/1988 at 5:42:08 pm and ending with the earthquake on 31/12/2012 at 23:48:30 h.

\subsection{Analysis of the Hurst Exponent Evolution Model}

Firstly, time series of seismic events is determined, for which the seismic catalog provided by the National Seismological Service, administered by the National Autonomous University of Mexico (UNAM) is used. ${ }^{30,31}$

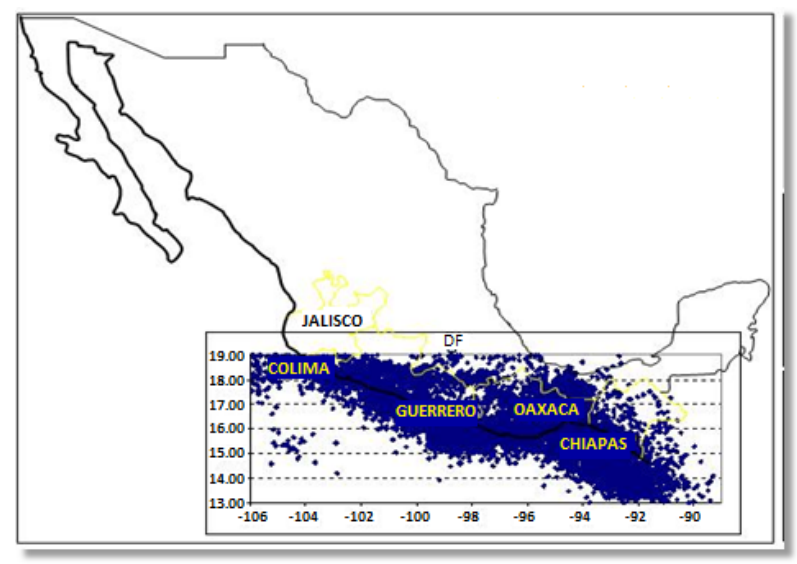

Fig. 2 Geographic location of the study area is shown in box delimiting the study window: Lengths $-89^{\circ}$ and $-106^{\circ}$ (W) and Latitudes $13^{\circ}$ and $19^{\circ}(\mathrm{N})$. Source: own.

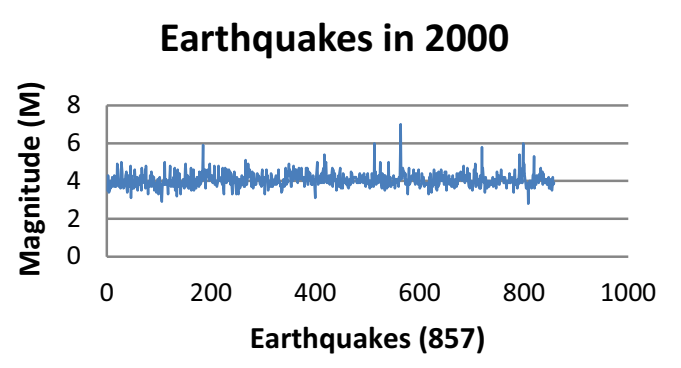

(b)

Fig. 3 Time series for seismic events that occurred in the Cocos Plate-Mexico for different year intervals as indicated in the title of the graphs $(\mathbf{d}, \mathbf{e}, \mathbf{f})$. Source: own. 
Earthquakes in 2011

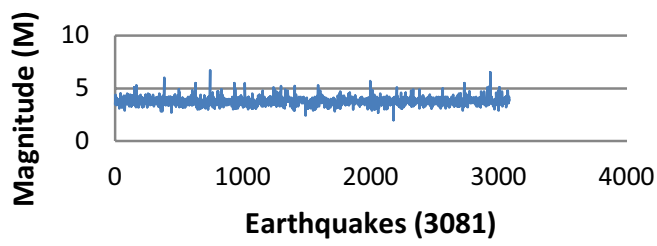

(c)

\section{Earthquakes from 1988-2000}

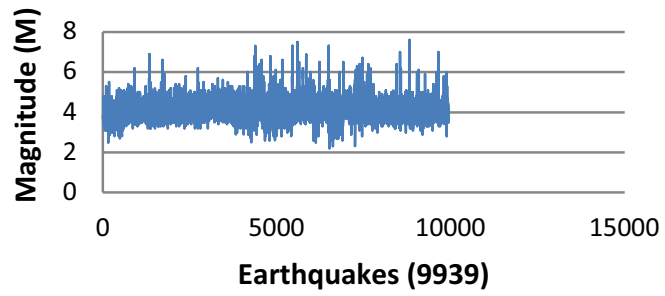

(e)
Earthquakes from 1988-1989

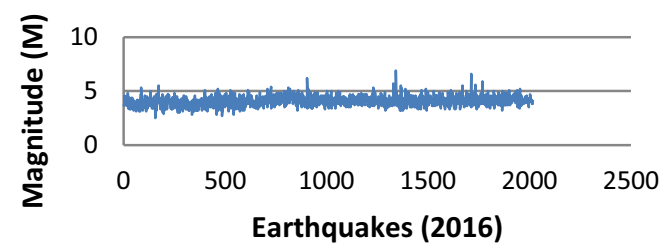

(d)

\section{Earthquakes from 1988-2011}

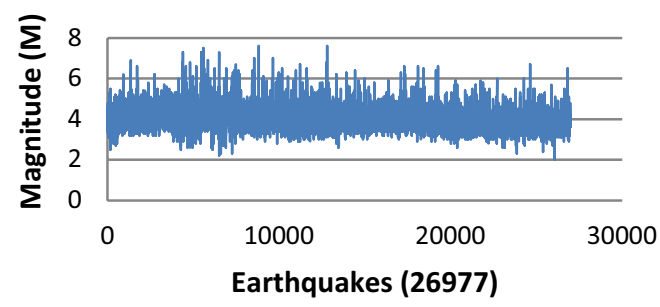

(f)

Fig. 3 (Continued)

Once filtered and prepared, the data were first divided the time series per year, for a period of 25 years, and then they were accumulated until reaching 25 years (see Fig. 3), then the files have to

\begin{tabular}{lllc}
\hline Year & $\begin{array}{c}\text { H R/S } \\
\text { Analysis }\end{array}$ & Interval & $\begin{array}{c}\text { H R/S } \\
\text { Analysis }\end{array}$ \\
\hline 1988 & 0.158 & 1988 & 0.158 \\
1989 & 0.164 & $1988-1989$ & 0.171 \\
1990 & 0.178 & $1988-1990$ & 0.171 \\
1991 & 0.168 & $1988-1991$ & 0.180 \\
1992 & 0.189 & $1988-1992$ & 0.168 \\
1993 & 0.226 & $1988-1993$ & 0.181 \\
1994 & 0.190 & $1988-1994$ & 0.166 \\
1995 & 0.222 & $1988-1995$ & 0.188 \\
1996 & 0.224 & $1988-1996$ & 0.189 \\
1997 & 0.201 & $1988-1997$ & 0.216 \\
1998 & 0.204 & $1988-1998$ & 0.213 \\
1999 & 0.256 & $1988-1999$ & 0.209 \\
2000 & 0.205 & $1988-2000$ & 0.203 \\
2001 & 0.212 & $1988-2001$ & 0.211 \\
2002 & 0.184 & $1988-2002$ & 0.201 \\
2003 & 0.201 & $1988-2003$ & 0.212 \\
2004 & 0.208 & $1988-2004$ & 0.207 \\
2005 & 0.182 & $1988-2005$ & 0.211 \\
2006 & 0.184 & $1988-2006$ & 0.203 \\
2007 & 0.134 & $1988-2007$ & 0.198 \\
2008 & 0.237 & $1988-2008$ & 0.204 \\
2009 & 0.200 & $1988-2009$ & 0.198 \\
2010 & 0.236 & $1988-2010$ & 0.212 \\
2011 & 0.272 & $1988-2011$ & 0.223 \\
2012 & 0.284 & $1988-2012$ & 0.227 \\
\hline & & &
\end{tabular}

be prepared to be analyzed by the rescaled range method using the software Benoit 1.3. ${ }^{32}$

With these time series, the files to be used in the software are prepared, and the Hurst exponent values obtained are shown in Fig. 4.

With the data obtained, the trends and distribution of the Hurts exponent values can be depicted as shown in Fig. 5.

Figure 5 shows the Hurts exponent evolution for the period considered, we can see the trends and behavior of the exponent over time, but unfortunately the seismic catalogs available are consistent from 1988. It will be desirable to pursue this type of analysis since talking geological time, 25 years represent only a small range, we think only provides a probability value perhaps little acceptable if they are to be used for prediction purposes.

\subsection{Hurst Exponent and Fractal Dimension}

The second analysis developed in this research, presents the possibility of finding of seismic activity relationship between the value found for the Hurst exponent and time series 3D fractal dimension, taking into account different threshold of magnitude.

So, to find the seismic activity 3D fractal dimension, the time series for events with magnitude greater or equal to $3^{\circ}$ on the Richter scale has to be defined, then increments of $0.5^{\circ}$ are considered until reaching $6^{\circ}$. 


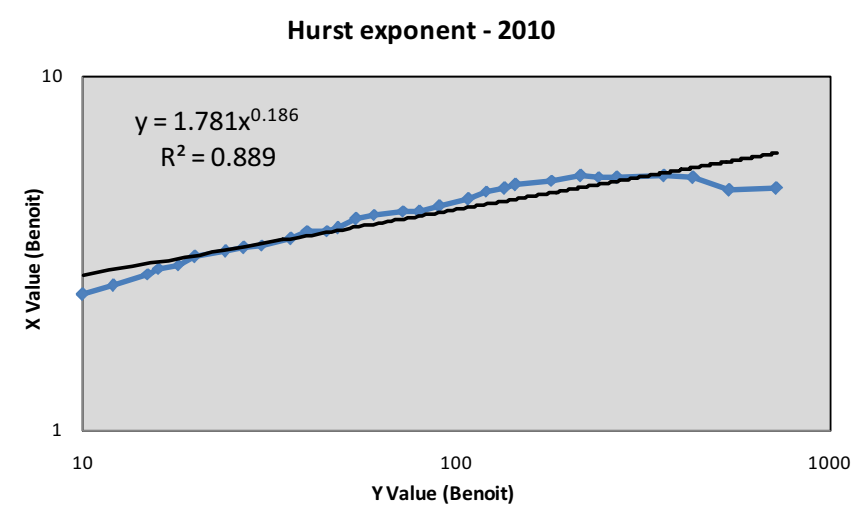

(a)

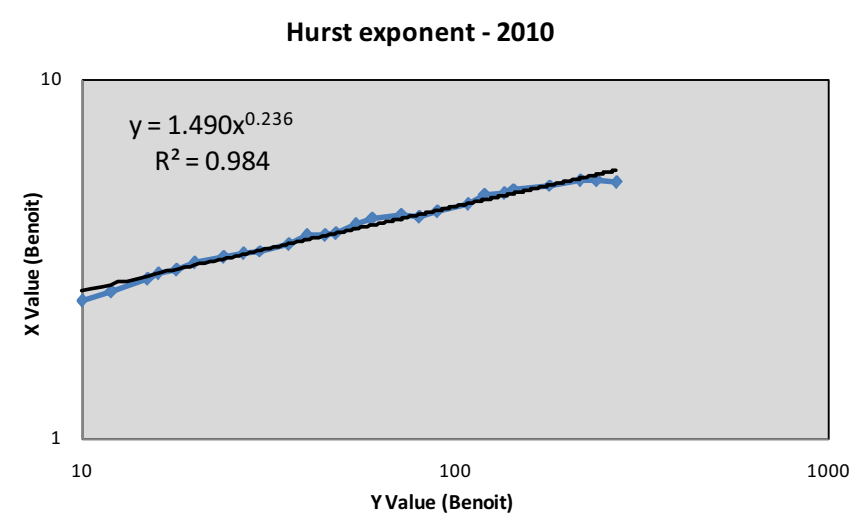

(b)

Fig. 4 Hurst exponent values calculated for seismic data. Table left to annual time series according to their magnitude (M) right table for cumulative time series for the interval indicated, also according to their magnitude $(M)$. The chart shows how the value was calculated Hurst exponent with Benoit ${ }^{\circledR} 1.3$ software with the method of Range Rescaling (R/S Analysis), for each of the 47 time series: (a) before adjustments and (b) after adjustment. Source: own.

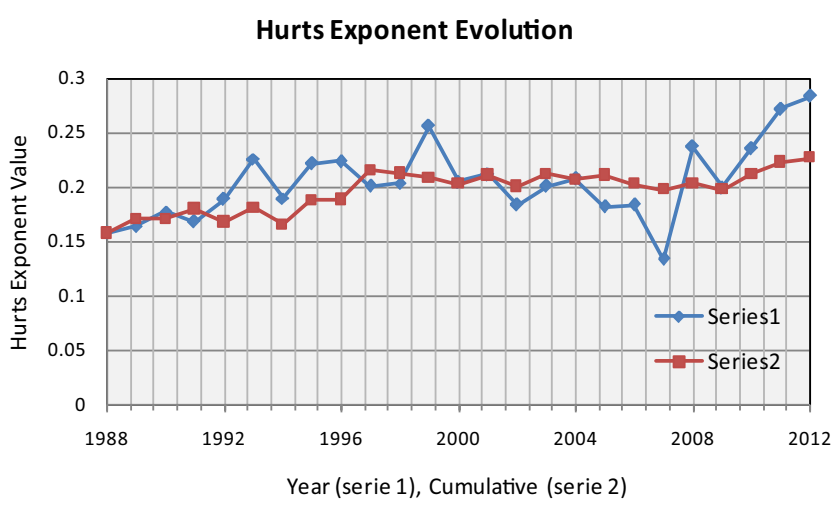

Fig. 5 Evolution model of the calculated values for the Hurst exponent of seismic activity occurred in the Cocos Plate-Mexico for a period of 25 years, from 1988-2012. Source: own.
The events density decreases each time because the number $N$ of earthquakes with a magnitude greater than $M$ occurs in a given time and space $m$ as a magnitude function with respect to the exponential distribution law which is the GutenbergRichter law, given in the following description:

$$
\log N=a-b M
$$

Once the magnitude threshold to be analyzed is determined, the needed data are prepared to find the fractal dimension using a computational program developed by the authors specifically for this application, which is based on the box dimension method (Box Dimension Estimation Method).

The box dimension is defined as the exponent $\mathrm{D}_{\mathrm{b}}$ in the relationship:

$$
N(d) \approx \frac{1}{d^{D_{s}}}
$$

With $N(d)$ is the number of lineal $d$ sized boxes required to cover a dataset distributed in a twodimensional plane. ${ }^{32}$

This method is based on the fact that for objects which are Euclidean, Eq. (8) defines the dimension. It requires a number of boxes proportional to $1 / d$ to cover a set of points on a thin line, proportional to $\left(1 / d^{2}\right)$ to cover a set of points uniformly distributed in a plane, $\left(1 / d^{3}\right)$ for three-dimensional space, and so on.

The dimension of boxes can be defined when the boxes are located at any position and orientation to minimize the number of boxes required to cover the set. Obviously, this is a complex computational problem, because the configuration that minimizes $N(d)$ has to be found among a great number of possibilities for covering the set with boxes of size $d$. For example, for 74 earthquakes of magnitude $M \geq 6$, in a space of $262,144\left(r_{7}=1 / 64,64^{3}\right)$ possibilities with a cubic dimension of $L=0.25^{\circ}$. This is because a power law as (8) is such that the exponent does not change if you multiply $N(d)$ or $d$ for any arbitrary constant. $^{32}$

In practice, to measure $D_{b}$, the number of linear size $d$ boxes needed to cover the whole range of values of $d$ is counted, and the logarithm of $N(d)$ is plotted on the vertical axis against the logarithm of $d$ in the horizontal axis. If the set is certainly fractal, this graph will follow a straight line with an inclination angle equal to $-D_{b}$. For evenly spaced points in the log-log space, it is better to choose boxes of size $d$ following a geometric progression 
$(d=1,2,4,8, \ldots)$ instead of using an arithmetic progression $(d=1,2,3,4, \ldots)$.

In theory, for each size box, the grid can be covered by a minimum number of boxes.

Figure 6 shows the density (number) of seismic events considered for different magnitude threshold.

With the necessary data files ready for processing, the software developed is executed, and the 3D fractal dimension for each threshold of magnitude is obtained. Notice that the time series of seismic event data are also analyzed, according to magnitude $M$.

This is needed to determine the Hurst exponent as mentioned above. Table 1 summarizes the data obtained from these analyzes.

The magnified threshold of magnitude $M \geq 6$ can be seen in Fig. 7 .

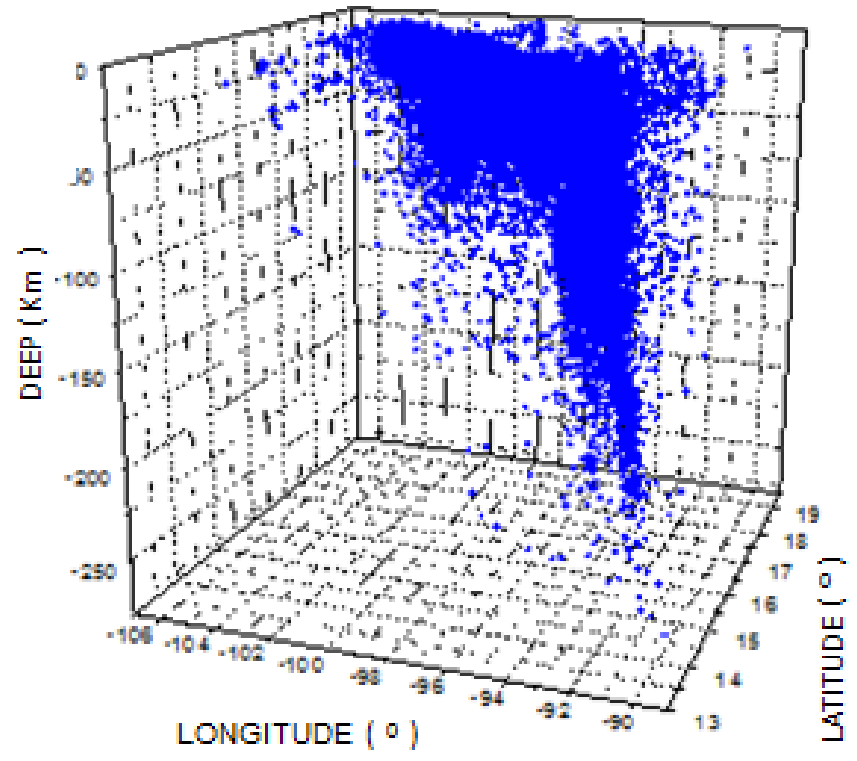

(a)

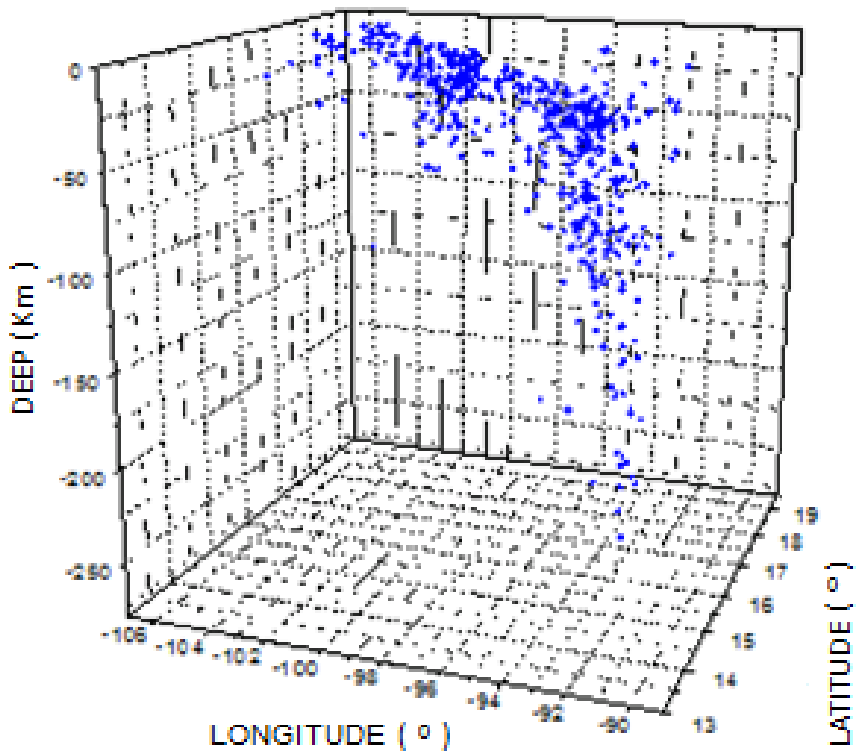

(c)

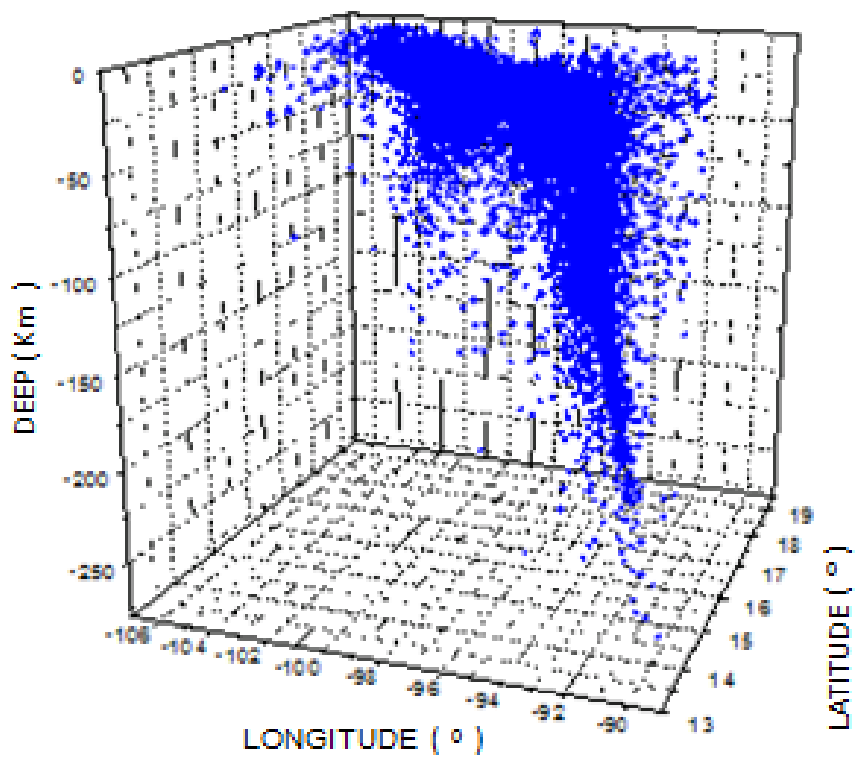

(b)

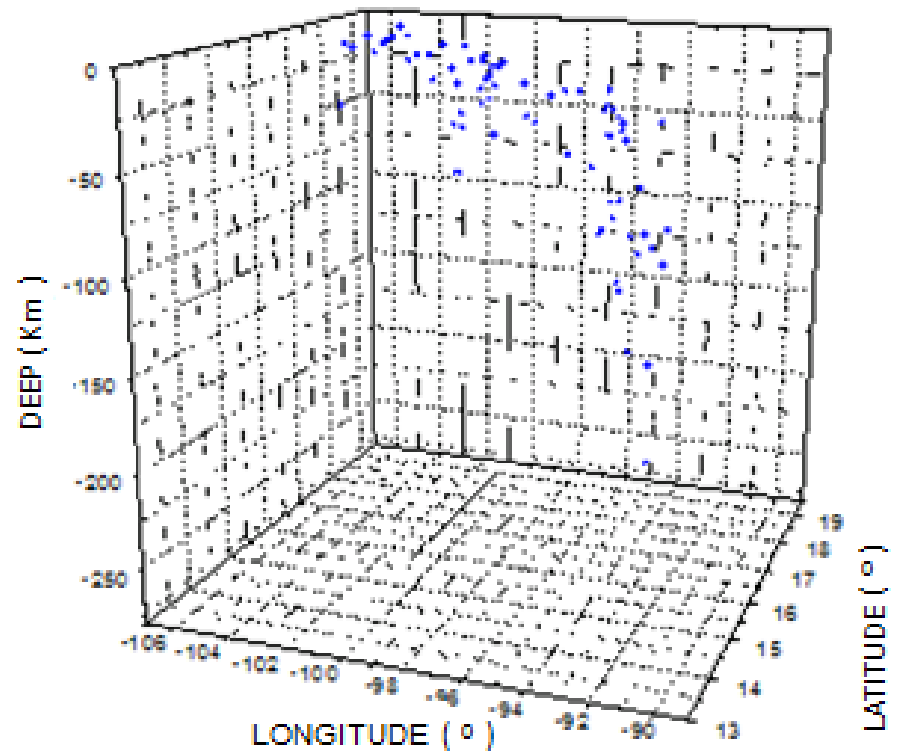

(d)

Fig. 6 Location of 3D seismic events (hypocenter) for different thresholds of magnitude occurred in the Cocos Plate-Mexico: (a) $M \geq 3$ with 31,276 earthquakes, (b) $M \geq 4$ with 13,866 earthquakes, (c) $M \geq 5,590$ earthquakes (d) $M \geq 6$, with 74 earthquakes. Note the decrease in seismic events (ratio Gutenberg-Richter) with increasing magnitude threshold $M$. For the period: 01/01/1988 to 31/12/2012. Source: own. 
Table 1 Values Obtained for the Fractal Dimension $\left(D_{f}\right)$ and Hurst Exponent $(H)$ of Seismic Events of Different Magnitude Threshold $(M)$.

\begin{tabular}{lccccccc}
\hline & \multicolumn{7}{c}{ Magnitude Threshold $\boldsymbol{M}$} \\
\cline { 2 - 9 } & $\boldsymbol{M} \geq \mathbf{3}$ & $\boldsymbol{M} \geq \mathbf{3 . 5}$ & $\boldsymbol{M} \geq \mathbf{4}$ & $\boldsymbol{M} \geq \mathbf{4 . 5}$ & $\boldsymbol{M} \geq \mathbf{5}$ & $\boldsymbol{M} \geq \mathbf{5 . 5}$ & $\boldsymbol{M} \geq \mathbf{6}$ \\
\hline Number of & & & & & & \\
$\quad$ earthquakes $(\boldsymbol{N})$ & 31,276 & 28,657 & 13,866 & 3,237 & 590 & 164 & 74 \\
Fractal Dimension $\left(\boldsymbol{D}_{\boldsymbol{f}}\right)$ & 2.137 & 2.133 & 2.073 & 1.874 & 1.575 & 1.249 & 1.054 \\
Hurst Exponent $(\boldsymbol{H})$ & 0.2153 & 0.2084 & 0.2146 & 0.2560 & 0.2635 & 0.2280 & 0.0940 \\
\hline
\end{tabular}

Notes: Magnitude $M$ since $\geq 3$ to $\geq 6$.

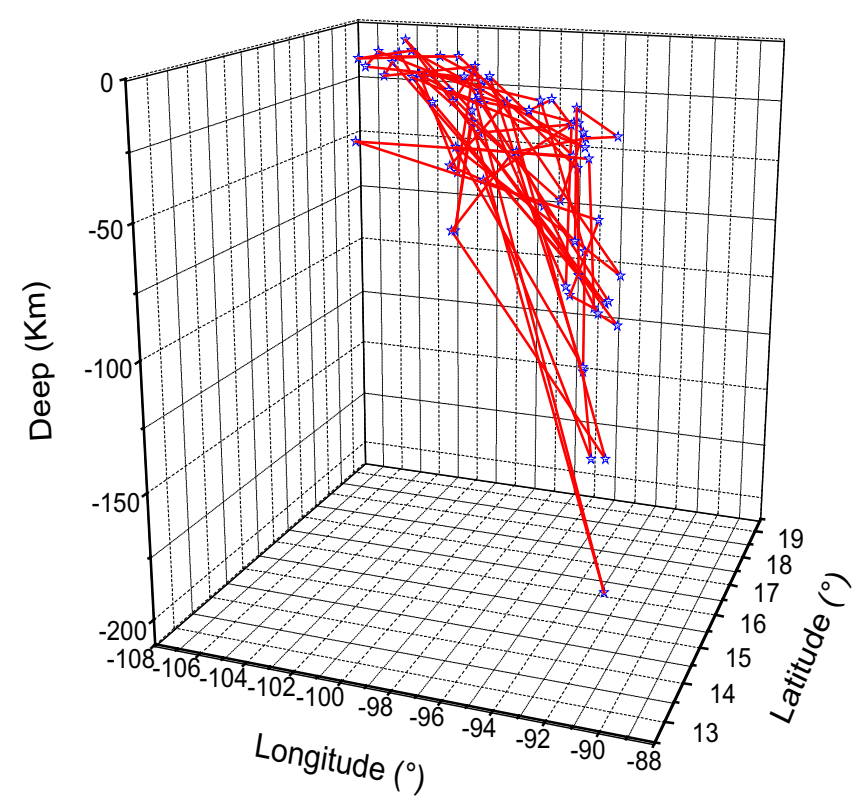

Fig. 7 Location 3D seismic events occurred in the Cocos Plate-Mexico, for a threshold of magnitude $M \geq 6$ and a period of 01/01/1988 to 31/12/2012 considered, with $N=74$ earthquakes. Source: own.

\begin{tabular}{lccc}
\hline Mag. $(\boldsymbol{M})$ & $\begin{array}{c}\text { Hurst } \\
\text { Exponen. } \\
(\boldsymbol{H})\end{array}$ & $\begin{array}{c}\text { Fractal } \\
\text { Dimension } \\
\left(\boldsymbol{D}_{\boldsymbol{f}}\right)\end{array}$ & $\begin{array}{c}\text { Corr. } \\
\text { Coeffi. } \\
(\boldsymbol{r})\end{array}$ \\
\hline 3 & 0.215 & 2.137 & - \\
3.5 & 0.208 & 2.133 & - \\
4 & 0.214 & 2.073 & -0.366 \\
4.5 & 0.256 & 1.874 & -0.974 \\
5 & 0.263 & 1.575 & -0.930 \\
5.5 & 0.228 & 1.249 & -0.502 \\
6 & 0.094 & 1.054 & 0.478 \\
\hline
\end{tabular}

When fractal dimension $\left(D_{f}\right)$ and the Hurst exponent $(H)$ are determined for different thresholds of magnitude $(M)$, according to Table 1 , then they are related by means of the correlation coefficient $\mathbf{r}$, which is shown in Fig. 8.

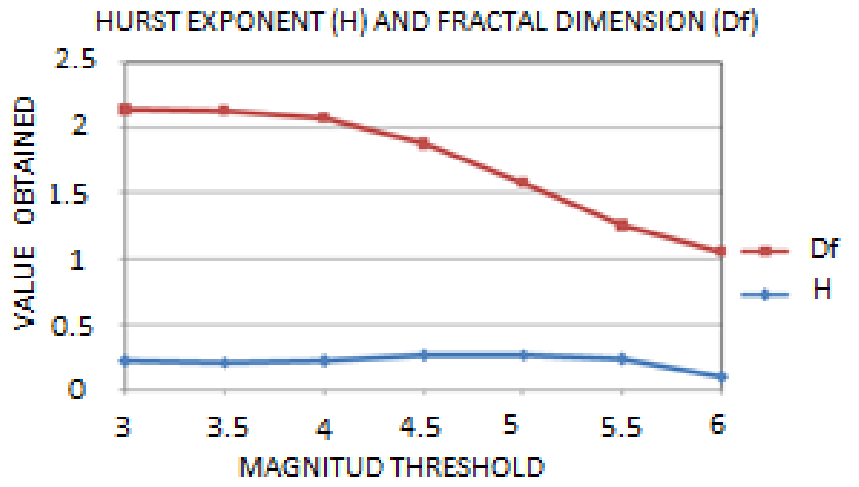

Fig. 8 Graphic correlation coefficient $(r)$ between the fractal dimension $\left(D_{f}\right)$ and Hurst exponent $(H)$, for seismic events that occurred in the Cocos Plate-Mexico, of different magnitude threshold $(M)$ for the reporting period 01/01/1988 to $31 / 12 / 2012$. Source: own.

\section{CONCLUSIONS}

As depicted in Fig. 5, there are differences between series 1 and 2. Series 1 (yearly) presents more inconsistent fluctuations and this has highs and lows causing the obtained peaks, while series 2 (cumulative) shows a uniform trend with some slight variations only for the years 92, 94 and 96. It can also be seen that most of the values for this graph, fall within the range from 0.15 to 0.25 for the value of the Hurst exponent. According to the obtained values, it can be seen that the graphic unfolds within the Hurst exponent values for $0<H<0.5$, indicating that the values of annual seismic activity remain in the range of anti-persistent even by accumulation, and the invariance with respect to scale exists associated with long-term with negative correlation. The fractal dimension of seismic events, especially for the case where $M \geq 6$, is close to 1 , since as already remarked, the space occupied by these seismic events practically defines a line (Fig. 7), which means that a cube of 262,144 points considered in the space may be occupied or not by one or more seismic events with a probability 
of 0.000267 occupancy for the iteration in which $L=0.25^{\circ}$. Finally, intermittence of the correlation coefficient between the Hurst exponent and seismic activity Fractal considered the last magnitude value observed, this is due to the involvement of large earthquakes which contribute significantly.

The scientific contribution of this paper is the novel way to analyze the dynamics of seismic phenomenon and have found that presents antipersistence through behavior in the evolution of Hurst exponent (Model) in this area of Mexico that has a high seismic activity. On the other hand, an analysis is developed to find whether there is any correlation between the evolution of Hurst exponent and fractal dimension 3D seismic activity occurred in southwestern Mexico, which will contribute to the understanding of how the seismic phenomenon through the considered variables (Hurst exponent and $D_{f}$ in $\left.3 \mathrm{D}\right)$, to advance the field of prediction. This opens a new field of research in geophysics, earthquakes, environmental, etc. processes, providing the possibility of developing cutting-edge research.

\section{ACKNOWLEDGMENTS}

The authors gratefully acknowledge the support of Conacyt and Instituto Politécnico Nacional through ESIME, and SIP project no. 200140728.

\section{REFERENCES}

1. B. Gutenberg and C. F. Richter, Bull. Seismol. Soc. Am. 34(4) (1944) 185-188.

2. B. Gutenberg and C. F. Richter, Seismicity of the Earth and Associated Phenomena, 2nd edn. (Princeton University Press, Princeton N. J., 2007).

3. D. L. Turcotte, Fractals and Chaos in Geology and Geophysics, 2nd edn. (Cambridge University Press, Cambridge UK, 1997).

4. D. L. Turcotte and G. Schubert, Geodynamics (Cambridge University Press, Cambridge U.K., 2002), pp. 9-49.

5. Y. Y. Kagan, Why does theoretical physics fail to explain and predict earthquake occurrence, Lect. Notes Phys. 705 (2006) 303-359.

6. A. Corral, Statistical features of earthquake temporal occurrence, Lect. Notes Phys. 705 (2006) 191221.

7. D. L. Turcotte, Scaling in geology: Landforms and earthquakes, Proc. Natl. Acad. Sci. USA 92 (1995) 6697-6704.

8. P. Bak et al., Unified scaling law for earthquakes, Phys. Rev. Lett. 88 (178501) (2002) 1-4.
9. A. Corral, Renormalization-group transformations and correlations of seismicity, Phys. Rev. Lett. 95 (2005) 028501.

10. A. Corral, Universal earthquake-occurrence jumps, correlations with time, and anomalous diffusion, Phys. Rev. Lett. 97 (2006) 178501.

11. K. Christensen, L. Danon, T. Scanlon and P. Bak, Unified scaling law for earthquakes, Proc. Natl. Acad. Sci. USA 99 (2002) 2509-2513.

12. M. Contadakis, Hydrologic changes as possible earthquake precursor in Greece, Nat. Hazards 23 (2001) 29-47.

13. L. Dajiong, Las nubes: un sismógrafo en el cielo. China Reconstruye, junio (1983) 35-37.

14. J. Derr, Earthquake lights: A review of observations and present theories, Bull. Seismol. Soc. Am. 63 (1973) 2177-2187.

15. E. Braun, Caos, fractales y cosas raras. La ciencia para todos, no. 150 (Fondo de cultura económica, México, 2003).

16. A. Balankin, Fractal behavior of complex systems, Científica 7(3) (2003) 109-128.

17. A. Balankin, Dynamic scaling approach to study time series fluctuations, Phys. Rev. E 76 (2007) 056120 .

18. A. Balankin et al., Scaling dynamics of seismic activity fluctuations, Europhys. Lett. 85 (2009) 1-6.

19. V. Talanquer, Fractus, Fracta, Fractal: Fractales, de laberintos y espejos. México: La ciencia para todos no. 147 (Fondo de cultura económica, México, 2003), pp. $43-61$.

20. C. Flores et al., The emergence of after-sales spare parts supply chain variability in a telecom firmA complex system approach, Fractals 20(1) (2012) $1-16$.

21. T. Vicsek, Fractal Growth Phenomena (World Scientific, 1989), pp. 1-6.

22. B. Mandelbrot, Fractals in Physics (Holland, Amsterdam, 1986), pp. 3-28.

23. S. Luo et al., Multiscale and fractal analysis of silicon content time series observed in blast furnace hot metal using Hurst exponent chain, Fractals 23(04) (2015) 1550036.

24. Yen-Ching, Introducing an interpolation method to efficiently implement an approximate maximum likelihood estimator for the Hurst exponent, Fractals 23(04) (2015) 1550045.

25. K. Chong, Fractal analysis on internet traffic time series. University of Fribourg, Switzerland, Econophysics Forum 3 (2003) arxiv: physics/0206012.

26. F. M. Ramos et al., Hurst exponent estimation of self-affine time series using quantile graphs, Physica A 444 (2016) 43-48.

27. E. Molino et al., Application of a time-scale local hurst exponent analysis to time series, Digital Signal Process. 37 (2015), 92-99. 
28. B. Mandelbrot and J. Wallis, Robustness of the rescaled range $\mathrm{R} / \mathrm{S}$ in the measurement of noncyclic long run statistical dependence, Water Resour. Res. 5 (1969) 967-988.

29. H. E. Hurst, Long-term storage capacity of reservoirs, Trans. Am. Soc. Civil Eng. 116 (1951) 770779 .
30. Servicio Sismológico Nacional, Reporte de sismos, sismo de Chiapas del 16 de enero de 2002, México: Instituto de Geofísica, UNAM. www.ssn.unam.mx.

31. Servicio Sismológico Nacional. UNAM, México. (2012).

32. BENOIT 1.3 W. Seffens, Science 285, Available at http://www.trusoft-international.com/(2007). 